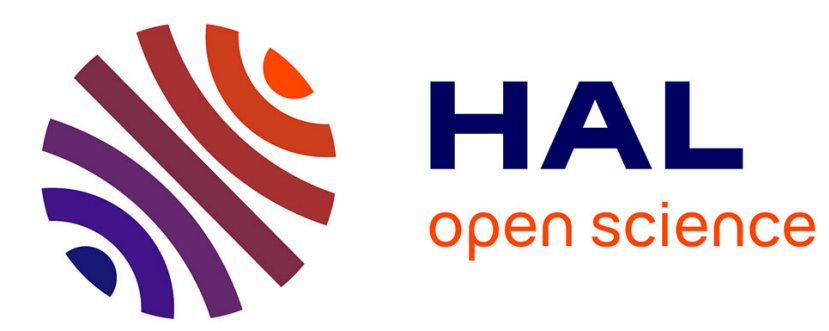

\title{
MR-Guided Thermotherapy of Abdominal Organs Using a Robust PCA-Based Motion Descriptor
}

Baudouin Denis de Senneville, Mario Ries, Grégory Maclair, Chrit Moonen

\section{To cite this version:}

Baudouin Denis de Senneville, Mario Ries, Grégory Maclair, Chrit Moonen. MR-Guided Thermotherapy of Abdominal Organs Using a Robust PCA-Based Motion Descriptor. IEEE Transactions on Medical Imaging, 2011, 30 (11), pp.1987-1995. 10.1109/TMI.2011.2161095 hal-01578171

\section{HAL Id: hal-01578171 \\ https://hal.science/hal-01578171}

Submitted on 28 Aug 2017

HAL is a multi-disciplinary open access archive for the deposit and dissemination of scientific research documents, whether they are published or not. The documents may come from teaching and research institutions in France or abroad, or from public or private research centers.
L'archive ouverte pluridisciplinaire HAL, est destinée au dépôt et à la diffusion de documents scientifiques de niveau recherche, publiés ou non, émanant des établissements d'enseignement et de recherche français ou étrangers, des laboratoires publics ou privés. 


\title{
MR-guided thermotherapy of abdominal organs using a robust PCA-based motion descriptor
}

\author{
Baudouin Denis de Senneville, Mario Ries, Grégory Maclair, and Chrit Moonen
}

\begin{abstract}
Thermotherapies can now be guided in real-time using magnetic resonance imaging (MRI). This technique is rapidly gaining importance in interventional therapies for abdominal organs such as liver and kidney. An accurate online estimation and characterization of organ displacement is mandatory to prevent misregistration and correct for motion related thermometry artifacts. In addition, when the ablation is performed with an extracorporal heating device such as High Intensity Focused Ultrasound (HIFU), the continuous estimation of the organ displacement is the basis for the dynamic adjustment of the focal point position to track the targeted pathological tissue.

In this paper, we describe the use of an optimized PCA-based motion descriptor to characterize in real-time the complex organ deformation during the therapy. The PCA was used to detect, in a preparative learning step, spatio-temporal coherences in the motion of the targeted organ. During hyperthermia, incoherent motion patterns could be discarded, which enabled improvements in motion estimation robustness, the compensation of motion related errors in thermal maps, and the adjustment of the beam position.

The suggested method was evaluated for a moving phantom, and tested in-vivo in the kidney and the liver of twelve healthy volunteers under free breathing conditions. The ability to perform a MR-guided thermotherapy in-vivo during HIFU intervention was finally demonstrated on a porcine kidney.
\end{abstract}

Index Terms-Image registration, Motion analysis, Motion compensation, Magnetic resonance imaging

\section{INTRODUCTION}

$\mathbf{M}$ INIMALLY-invasive interventional procedures such as tissue ablation with radio-frequency (RF) and noninvasive ablation techniques such as high-intensity focused ultrasound (HIFU) show a high potential in oncology and cardiology as an alternative to classical surgery [1] [2]. The objective is a necrosis of the tissue caused by an elevated thermal dose due to sustained heating [3]. These methods are thus ideally paired with a non-invasive imaging modality which can provide both temperature measurements and target tracking information. The most promising candidate for this role is real-time MR-thermometry based on the water proton resonance frequency (PRF) [4]. This technique provides continuous temperature mapping inside the human body as well as target tracking information by exploiting the complex nature of the MR-signal: whereas the signal magnitude provides anatomical information such as the position and the composition of the tissue, the phase $\varphi$ is directly proportional

Baudouin Denis de Senneville, Mario Ries and Chrit Moonen are with the Laboratory for Molecular and Functional Imaging: From Physiology to Therapy, FRE $3313 \mathrm{CNRS/University} \mathrm{of} \mathrm{Bordeaux} \mathrm{2,} 33076$ Bordeaux, France, (e-mail: \{baudouin,gregory,ries,moonen\}@imf.u-bordeaux2.fr).

Manuscript received ...; revised ... to the local magnetic field $\vec{B}$ and to the local proton resonance frequency (and thus the local temperature). The simplest way to obtain an estimate of the temperature changes at instant $n$ (noted $\Delta T_{n}$ ) is to evaluate phase shifts between dynamically acquired phase images and reference data sets as follows:

$$
\Delta T_{n}=\left(\varphi_{\text {ref }}-\varphi_{n}\right) \cdot k \quad k=\frac{1}{\gamma \cdot \alpha \cdot \vec{B} \cdot T E}
$$

where $\gamma$ is the gyromagnetic ratio $(\approx 42.58 \mathrm{MHz} /$ Tesla), $\alpha$ the temperature coefficient $(\approx 0.009 \mathrm{ppm} / \mathrm{K})[5]$, and $T E$ the echo time.

This new type of interventional procedure is very interesting for treatment of vital organs (kidney, liver, heart). However those organs are subject to physiological motion which leads to the following challenges:

1) Misregistration between phase images must be compensated, and temperature information must be mapped to a reference position of observed organs to obtain thermaldose measurements, requiring the voxel-by-voxel temporal integration of the temperature.

2) Reliable temperature measurement using PRF based MR-thermometry on abdominal organs is complicated by the fact that the target moves through an inhomogeneous and time-variant magnetic field, causing strong thermometry artifacts [6].

3) The estimation of the organ displacement is the basis for the dynamic adjustment of the HIFU beam to track the targeted pathological tissue, preventing an inefficient treatment and unwanted destruction of healthy tissue [7].

To accomplish these tasks, the organ motion must be estimated and a number of techniques have been proposed in the past for various imaging modalities [8] [9]. In the context of real-time guidance of the hyperthermia, navigator echoes [10] or ultrasonic echoes have been originally proposed. However, only translational displacements can in those cases be estimated. Since modern MRI acquisition methods allow the rapid acquisition of large data volumes combined with an excellent tissue contrast and high spatial resolution, complex deformation can be estimated on a voxel-by-voxel basis using optical flow based approaches [11].

Combined with the motion estimation process, several correction strategies have been proposed in the past to correct for thermometry artifacts, such as navigator echoes [10] or referenceless phase corrections [12]. An approach emerged recently which consists of analyzing phase perturbation with motion during a pre-treatment step performed prior to the intervention [7]. For this purpose, a reference data-set of 
magnitude and phase images is recorded during the motion cycle of the organ. These images are used to evaluate a direct linear relation between the target motion and resulting phase variations.

Finally, since many applications in the field of MR-guided interventions also exploit the MR-image information for a direct retroactive control of the heating device [7], both the update time and the latency of the obtained motion field are key points: for typical abdominal organ motion, it has been recently demonstrated that a minimal frame rate of 10 images/second is required and the latency (i.e the delay between the actual time of displacement and the availability of the motion information) must not exceed 100 milliseconds [13]. This latency is composed by the sum of the remaining acquisition time after echo-formation, the required data transport time, the image processing time, and the switching time of the HIFU-generator.

Black et al. proposed in 1997 a robust image-based motion descriptor (i.e. a small set of parameters representing the deformation) evaluated using a reduced parameterized flow models initially computed, during a preparative learning step, with a Principal Component Analysis (PCA) [14]. The technique was originally evaluated in the scope of human labial recognition. Recently, Mauldin et al. proposed a PCA-based displacement estimation in the context of ultrasound imaging [15]. Previous work of the authors' group [16] [17] explored the possibility to apply the PCA-based motion descriptor for real-time MR-guided thermotherapy of mobile organs. The PCA-based motion descriptor was used to detect, in a preparative learning step, spatio-temporal coherences of the periodic organ motion. During hyperthermia, incoherent motion patterns could be discarded, which allowed the following improvements:

1) Optical-flow based algorithms rely on the assumption of conservation of local intensity along the trajectory which can be violated during thermotherapy because rapid MRimaging is in general associated with low Signal-ToNoise ratio (SNR). In addition, since the tissue is heated, several MR relevant tissue properties such as $T_{1}, T_{2}$ and $T_{2}^{*}$ relaxation times are subject to change during imaging [18]. This leads to local intensity variations, which in turn can be misinterpreted by optical-flow based algorithms as "motion". The PCA-based motion descriptor was used to provide a flow field that was consistent with the learned model and robust under the assumption of global brightness constancy but allowed local intensity variations.

2) The PCA-based motion descriptor was used to model the magnetic field variation with the target displacement. This improved the correction of motion related errors in temperature maps.

In the current paper, this PCA-based method was modified as follows: We first briefly introduce a method, based on the PCA-based representation, which is designed to separate physiological displacement from noise contributions. This step was found to be a necessary prerequisite for the practical application of the method. The potential applications enabled by the technique are then described and tested with a phantom experiment, and an in-vivo thermometry stability study in the kidney and the liver of twelve healthy volunteers under free breathing conditions. The ability to perform a MR-guided thermotherapy in-vivo during a real intervention was finally demonstrated on a porcine kidney during a HIFU heating experiment.

\section{METHOD DESCRIPTION}

During the therapy, MRI is used for two tasks: MR thermometry and target tracking. Since the basis of the PCAbased approach is described in detail in [16] [17], only a short summary is given here. Details are given for the method designed to separate physiological displacement from noise contributions, based on the PCA-based representation.

We evaluated the method in 2D since it is in practice possible for respiratory induced motion, to choose the imaging plane direction parallel to the principal axis of the organ displacement.

\section{A. Preparative learning step}

Motion patterns were learned during a preparative learning step performed before hyperthermia, based on a training set of $N$ images (magnitude and phase). The motion cycle was sampled with a sufficient density in order to avoid discretization errors. With a typical imaging frame rate of 10 images per second and a respiration frequency of 3-5 seconds this pretreatment step can be completed in a relatively short duration of 15-20 seconds (in this study, we used $N=200$ images).

1) Training set of organ displacement: A model of image motion was obtained from a training set of $N$ flow fields. For that purpose, an affine transformation was first estimated using a differential Gauss-Newton approach [19]. The obtained motion field was used for preconditioning of a more complex deformation estimation: The implemented approach was originally proposed by Horn\&Schunck [20] for optical image stabilization and computed a displacement field on a pixel-by-pixel basis. It includes a modification proposed by Cornelius\&Kanade [21], which relaxes the condition of intensity conservation from a global to a regional conservation. Finally, in order to optimize the computation time and to stabilize the convergence of the algorithm, a multi-resolution scheme was used [22] which iterated the registration algorithm from a four-fold down-sampled image step-by-step to the full image resolution.

2) Learning parameterized flow models: At this point, a model of image motion was obtained, which was a set of $N$ optical flow fields. The set was used to build a parameterized flow model. PCA was used to find an orthonormal basis that spans an $N$-dimensional vector space and depict the underlying characteristic patterns of the motion cycle.

3) Estimation of the motion descriptor: The periodic motion cycle was characterized with a small set of parameters as follows: The approximated spatial transformation $T_{t}$ was, for each pixel $(x, y)$ calculated as a linear combination of the previously computed basis vectors $B_{i}$ :

$$
T_{t}(x, y)=\sum_{i=0}^{M-1} D_{i}^{t} B_{i}(x, y)
$$


where $D_{i}^{t}(0 \leq i<M)$ was the motion descriptor (i.e. a set of parameters representing organ displacement), $M(M \leq N)$ the size of the motion descriptor, and $0 \leq t<N$ was the index of each image of the training set. The objective was thus to find the coefficients $D_{i}^{t}$ that produced a flow field minimizing the matching error:

$$
L S=\left(M_{r e f}-T_{t}^{-1}\left(M_{t}\right)\right)^{2}
$$

where $M_{r e f}$ was the reference magnitude image and $M_{t}$ the actual image. This minimization was realized using a Marquardt-Levenberg least square $(L S)$ solver [23]. To stabilize the convergence of the algorithm, a spatial Gaussian filter (kernel $3 \times 3$ ) was applied on the new incoming image $M_{t}$, and the motion descriptor obtained from the previous acquisition was used as a starting point for the new motion estimation.

4) Separation of physiological displacements from noise contributions: We used the PCA-based representation of the motion to detect spatio-temporal coherences of the periodic organ motion, in order to separate physiological displacements from noise contributions. For that purpose, only the $M$ eigenvectors $B_{i}$ associated with the $m$ largest eigenvalues $\lambda_{i}(0 \leq$ $i<M<N)$ were conserved as follows. The objective was thus to determine the threshold value for $M$, which separates eigenvectors representing physiological motions $(i \leq M)$ and from eigenvectors coding for noise contribution $(i>M)$. To accomplish this task, possible values for $M$ were iteratively enumerated from 2 to $N$ (we note $m$ the iteration number), and, for each $m$, the following test was performed:

1) The PCA-based motion descriptors $D_{i}^{t}$ were computed for all acquisitions of the preparative learning step.

2) A temporal variation of the $m^{t h}$ coefficient of the motion descriptor (i.e. $D_{m-1}^{t}$ ) was analyzed over the preparative learning step, as follows. Since the respiratory and the cardiac activities are periodic, we analyzed $D_{m-1}^{t}, \forall t$, $0 \leq t<N$ in the Fourier domain. Typical periods of the respiratory and cardiac activities are, in the general case, in the range of $3-6$ seconds and $0.5-2$ seconds, respectively. A threshold of $4 \mathrm{~Hz}$ was used to separate physiological motions from the noise contribution, and the maximal spectral magnitude was compared for lower and higher frequencies. The corresponding $(m-1)^{t h}$ eigenvector was considered to be noise corrupted if the spectral magnitude for frequencies above $4 \mathrm{~Hz}$ exceeds the value of $20 \%$ of the main peak below $4 \mathrm{~Hz} . M$ was then set to $(m-1)$, and the iterative enumeration was stopped.

5) Modelling motion induced phase changes: The $N$ images of the preparative learning step were used to evaluate the linear relation between organ motion and phase variation. For that purpose, the obtained motion field was used to remap all pixels of the phase images to their reference position. The overall magnetic field variations were approximated as the sum of linear phase changes of each principal motion component on a pixel-by-pixel basis:

$$
T_{t}^{-1}\left(\varphi_{t}(x, y)\right)=\sum_{i=0}^{M-1} D_{i}^{t} P_{i}(x, y)+P_{M}(x, y), 0 \leq t<N
$$

where $P_{i}(0 \leq i<M)$ denotes the parameterized magnetic field model, with $P_{M}$ representing the initial phase distribution, and $D_{i}^{t}$ were the coefficients calculated from Eq. (2) and (3). From the set of $N$ equations with $m$ unknowns defined by (4), an overestimated system could be obtained if $m \ll N$. $P_{i}(x, y)$ are individually computed for each pixel $(x, y)$ using a Singular Value Decomposition (SVD).

\section{B. Hyperthermia procedure}

During hyperthermia, each time a new image was acquired, the PCA-based motion descriptor was estimated and used to compute (with Eq. (3)) a flow field that was consistent with the learned model. The PCA-based motion descriptor was then used to correct for motion related errors on temperature maps as follows: During hyperthermia, the parameterized magnetic field model $P_{i}$ allows the reconstruction of magnetic field distribution corresponding to the current position of an organ. A synthetized reference non-heated phase image was calculated using the motion descriptor $D_{i}^{t}$ representing the actual organ displacement as follows:

$$
\varphi_{r e f}(x, y)=\sum_{i=0}^{M-1} D_{i}^{t} P_{i}(x, y)+P_{M}(x, y)
$$

In this study, we set an upper bound of 6 for $M$, in order to keep the computational time for the motion estimation process of one image below $25 \mathrm{~ms}$ with the used experimental setup.

The measured registered phase image was subtracted to the reference phase map $\varphi_{\text {ref }}$ to compute a motion corrected temperature map (with Eq. (1)). Since the $2 \pi$ periodicity of the image phase could lead to aliasing artifacts in the temperature maps, a temporal phase unwrapping on a pixel-by-pixel basis was applied. This process was valid under the condition that the temperature variation between two successive acquisitions did not create a phase variation greater than $\pi$.

\section{Experimental setup}

Dynamic MR temperature imaging was performed on a Philips Achieva 1.5 T (Philips Healthcare, Best, The Netherlands). MR images were processed using a dual processor (INTEL $3.1 \mathrm{GHz}$ Penryn, four cores) workstation with $8 \mathrm{~GB}$ of RAM and dual $1 \mathrm{~GB} / \mathrm{s}$ network interface cards.

Three experiments were performed to assess the improvement obtained with the PCA-based motion descriptor:

1) The method was applied on an ex-vivo heated phantom prone to a periodical translational displacement. The estimated motion was compared to reference positions provided by an external sensor.

2) The potential of the proposed method to modelize the magnetic field perturbation and to improve the correction for motion related errors on thermal maps was evaluated 
on the abdomen of 12 healthy human volunteers under free-breathing conditions.

3) A real-time tracked HIFU heating experiment was performed on a porcine kidney. Since the kidney displacement was rigid, the target motion could be succesfully estimated on-line by a translation restrained on a ROI manually set on the kidney at the begining of the hyperthermia (a differential Gauss-Newton approach was used [19]). The estimated displacements were subsequently used as reference to evaluate off-line the proposed PCAbased method.

It is important to see those proposed experiments in the appropriate context: Our PCA-based method presents a real interest only for elastic organ deformation such as in the liver, and was not a necessary prerequisite for experiments \#1 and \#3. However, those two experiments were designed to quantify, using reference positions, the accuracy and the precision of the proposed PCA-based method under thermoablation conditions.

1) Ex-vivo heating study: 3000 images were dynamically acquired during 3 minutes using a dual-shot, gradient recalled echo-planar acquisition sequence with the following parameters: repetition time $(T R)=30 \mathrm{~ms}, T E=15 \mathrm{~ms}$, bandwidth in readout direction $=1777 \mathrm{~Hz}$,flip angle $=20^{\circ}$, field of view $(F O V)=256 \times 104 \mathrm{~mm}^{2}$, slice thickness $=5 \mathrm{~mm}$, matrix $=128 \times$ 58. A porcine muscle was positioned on a motorized plateform, which generated a periodic translational displacement (amplitude $=10$ pixels and frequency $=0.5 \mathrm{~Hz}$ ). RF heating was performed 10 seconds after the end of the preparative learning step, using a clinical MR-compatible bipolar RF device (Radionics, Burlington, MA) with $8 \mathrm{~W}$ of RF-power during 75 seconds. For an independent assessment of the object displacement, an additional navigator echo, which provided a one dimensional displacement information $(0.5 \mathrm{~mm}$ precision $)$, was positioned parallel to the displacement on the apex of the muscle in order to get the reference displacement. We compared temperature maps obtained when phase images of Eq. (5) were registered with the navigator echo (which serves as reference results), with the Cornelius\&Kanade algorithm (the algorithm is described in details in section II.A.1) and with the PCA-based motion descriptor.

2) On-line in-vivo study: Dynamic MRI was performed under free-breathing conditions on the abdomen of 12 healthy human volunteers under real-time conditions. An imaging frame rate of $10 \mathrm{images} / \mathrm{s}$ was maintained for five minutes of MR-imaging while MR-Thermometry was performed in real-time. The MR sequence was a single-shot gradient recalled echo-planar which employed the following parameters: 3000 dynamic coronal images, one slice, $T R=100 \mathrm{~ms}$, $T E=26 \mathrm{~ms}$, bandwidth in readout direction $=2085 \mathrm{~Hz}$, flip angle $=35^{\circ}, F O V=256 \times 168 \mathrm{~mm}^{2}$, slice thickness $=6 \mathrm{~mm}$, matrix $=128 \times 84$, using a four element phased array body coil. No hyperthermia was performed and the statistical evaluation of the temperature stability was performed on the kidney and the liver of each volunteer individually by averaging the temporal temperature standard deviation over a manually defined ROI. The thermometry was compared with a synthetized reference non-heated phase expressed in Eq. (4) and (5), as the linear combination of the six free parameters of an affine transformation [11] (a differntial Gauss-newton approach was used for that purpose).

3) In-vivo heating study on a porcine kidney: MRI guided HIFU was performed in vivo in the kidney of a pig under general anesthesia and artificial breething. An imaging frame rate of 10 images/s was maintained for 2.5 minutes using a single-shot gradient recalled echo-planar sequence. The MR sequence employed the following parameters: 1500 dynamic sagittal images, one slice, $T R=100 \mathrm{~ms}, T E=41 \mathrm{~ms}$, bandwidth in readout direction $=2085 \mathrm{~Hz}$, flip angle $=35^{\circ}, F O V=320 \times 140$ $\mathrm{mm}^{2}$, slice thickness $=6 \mathrm{~mm}$, matrix $=128 \times 56$, using the integrated three elements phased array coil of the HIFU system. A MR compatible Philips HIFU ablation system (Sonalleve, Philips Healthcare, Helskinki, Finland) composed of a table top containing a 256 elements HIFU transducer, integrated in the $1.5 \mathrm{~T}$ Achieva-Intera MRI was used to perform a temperature elevation. The transducer radius and aperture were $120 \mathrm{~mm}$ and $126 \mathrm{~mm}$, respectively, creating an ellipsoid focal point $\left(1 \times 1 \times 7 \mathrm{~mm}^{3}\right)$. The animals were placed in right lateral decubitus position so that the kidney was accessible through an unobstructed beam-path directly below the rib-cage. Motion compensated MR-thermometry and tracking was performed for a duration of 1 minutes on the kidney with power of 100 W. A temporal filtering based on an infinite impulse-response filter was applied to the temperature maps to increase the SNR (more details can be found in [11]).

\section{RESULTS}

\section{A. Ex-vivo heating experiment}

Fig. 1 shows thermal maps obtained after 50 seconds of RF heating obtained using the reference navigator echo based registration (1a), the Cornelius\&Kanade algorithm (1b) (note the deformation of the heated area), and the PCA-based motion descriptor are displayed (1c). A detail of the anatomical image in the heated area before hyperthermia and an image obtained after 50 seconds of RF heating are given in Fig. 1a. The position of each electrode of the bipolar RF device is indicated with the two red arrows. A strong signal decrease can be observed during the hyperthermia process in the heated area (see green arrow) due to a large temperature change. It can be observed that the PCA-based motion descriptor provided temperature maps which were similar to the reference results.

An analysis of the estimated motion field accuracy over the whole duration of the experiment is presented in Fig. 2a. For that purpose, the euclidean error between the estimated displacement and the navigator echo information was computed in a pixel located in the heated area (reported by the green arrow in Fig. 1a). It can be observed in the first 10 seconds (i.e. before the energy deposition) that the precision was comparable with the PCA-based registration. However, the Cornelius\&Kanade approach provided poor results during hyperthermia procedure starts (up to 6 millimeters of error), while the PCA-based method remained stable and accurate over the time (less than 2 millimeters of error). Fig. 2 also shows the temporal evolution of the temperature in the same pixel with the Cornelius\&Kanade registration method 


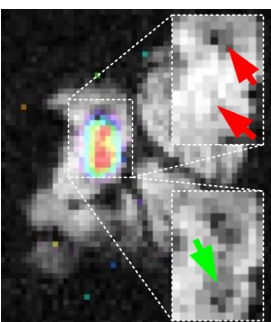

(a)

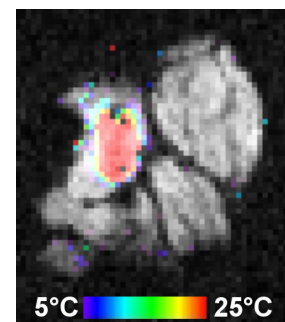

(b)

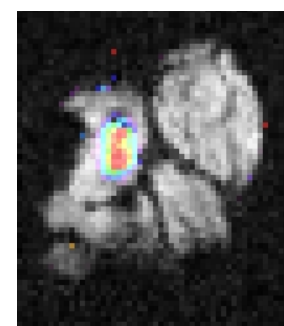

(c)
Fig. 1: Thermometry results obtained for the ex-vivo RF heating experiment. The computed temperature maps after 50 seconds of heating are displayed when the displacement information was obtained from the navigator echo (a), the Cornelius\&Kanade approach (b) and the PCA-based motion descriptor (c). The position of each electrode of the bipolar $\mathrm{RF}$ device is indicated by the two red arrows in the first insert, which displays the targeted region on an image aquired before hyperthermia. The second insert shows the strong signal decrease observed in the same area during hyperthermia due to the large temperature change (see green arrow).

(2b), and with the PCA-based registration (2c). Compared to the reference navigator echo results (red line), the temperature measured using the Cornelius\&Kanade registration was overestimated during the heating process. Contrary to the implemented Cornelius\&Kanade approach, the temperature evolution obtained with the PCA-based motion descriptor was close to the reference results obtained with the navigator echo.

\section{B. On-line in-vivo study}

The volunteers studies depicted various conditions: Over the twelve volunteers, the SNR was evaluated as $10 \pm 2.5$ $(\min =7, \max =14)$ in the kidney and $7 \pm 3(\min =4, \max =14)$ in the liver. The peak-to-peak motion amplitude obtained from the landmark points was $10 \mathrm{~mm} \pm 4.5$ (minimum $=4$, maximum=18) in the kidney and $11 \mathrm{~mm} \pm 4.5$ (minimum $=6$, maximum $=18$ ) in the liver. Due to variations of the respiratory volume, the peak-to-peak amplitude increased between the preparative learning step and the interventional procedure in patients \#8, \#9 and \#12 (where an increase of 50 to $65 \%$ was measured). This value exceeded $150 \%$ in patient \#7. Less than $10 \%$ of increase was measured in patients \#2, \#3, \#5 and \#10.

Fig. 3 details the improvement of the temperature precision when the PCA-based motion descriptor was used to characterize the organ deformation in Eq. (4) and (5), as compared to an affine motion model. In average over all volunteers, the temperature stability was improved from an initial value of over $2.3{ }^{\circ} \mathrm{C}$ to $2{ }^{\circ} \mathrm{C}$ (kidney) and $3.3{ }^{\circ} \mathrm{C}$ to $2.7{ }^{\circ} \mathrm{C}$ (liver) using the PCA-based motion descriptor. None of the data were rejected due to misregistration or faulty phase correction due to the absence of spontaneous motion.

Fig. 4 and 5 detail results obtained in the volunteer for which the most significant motion amplitude variation was detected between the preparative learning step and the hyperthermia procedure (i.e. volunteer \#7). Fig. 4 shows the

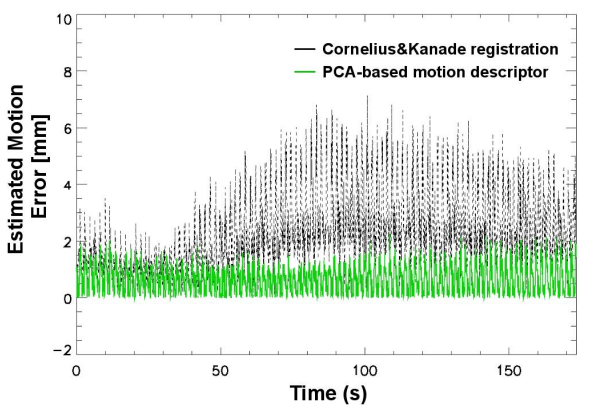

(a)

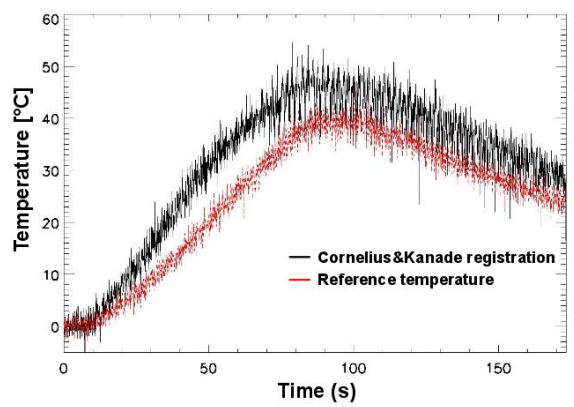

(b)

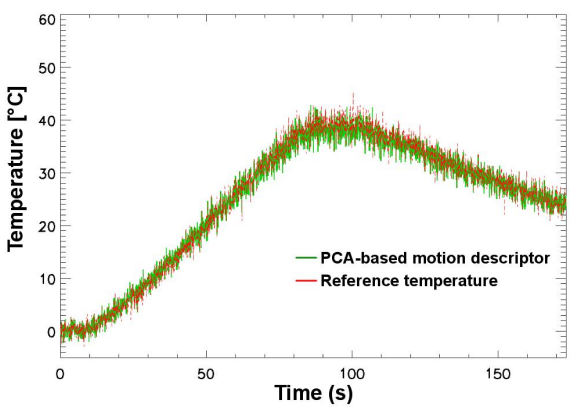

(c)

Fig. 2: (a): Temporal analysis of the registration accuracy in a pixel located in the heated area (green arrow on Fig. 1a) obtained with the Cornelius\&Kanade algorithm (black line) and the PCA-based motion descriptor (green line). (b): Temporal evolution of the temperature obtained with phase images registered with the Cornelius\&Kanade algorithm. (c): Temporal evolution of the temperature obtained with phase images registered with the PCA-based motion descriptor. The reference temperature obtained with navigator echo based displacements is displayed with the red line in (b) and (c).

vertical displacement estimated during the preparative learning step (4a) and during hyperthermia (4b) using the PCA-based registration (solid line). Manually tracked results provided by a staff scientist with a precision of a pixel (red dots) in a landmark located in the liver are also reported for comparison (red arrow in Fig. 5c). Due to deep variations of the breething, an increase of the peak-to-peak amplitude by $150 \%$ can be observed between the preparative learning step and the interventional procedure in this case. Even in this case, the proposed correction ensured $2{ }^{\circ} \mathrm{C}$ of temperature stability in $70 \%$ of all pixels of both the kidney and the liver, as shown in Fig. 5b. The first and the fourth eigenvectors are displayed in 
Fig. 5c and 5d, respectively (for each pixel the amplitude of the displacement vector was computed. Each map was normalized between 0 and 1 for an easier vizualisation). Each of these eigenvectors represented a physiological component of the organ deformation: while the first component modelized the respiratory motion (motion field vectors were oriented in the head-foot direction), the fourth component represent artereal pulsations caused by the cardiac cycle. The estimation of a complex deformation was mandatory in the liver where the motion amplitude varied with a factor two (see Fig. 5c). (a)

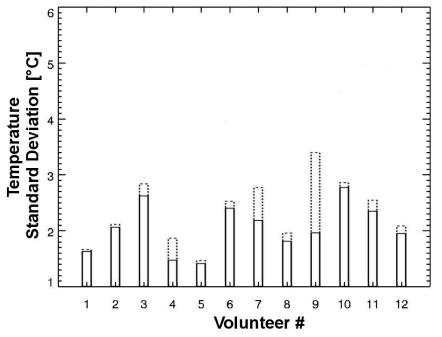

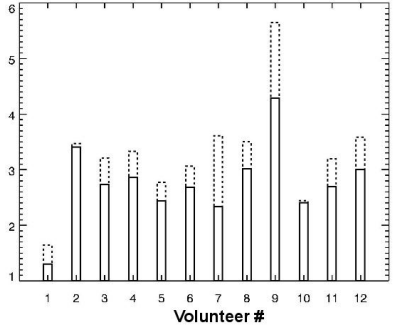

(b)
Fig. 3: Histogram of the temperature precision obtained over the twelve volunteers in the kidney (a) and the liver (b), when the temperature maps were computed using an affine motion model (dash line) and using the PCA-based motion descriptor (solid line).

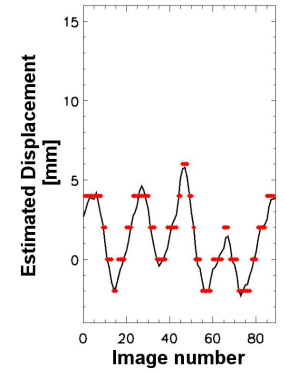

(a)

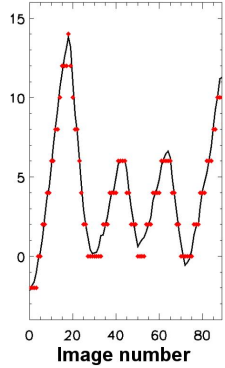

(b)
Fig. 4: Comparison of the displacement estimated in the headfoot direction using the PCA-based registration (solid line) and manually tracked by a staff scientist with a precision of a pixel (red dots) in a landmark located in the liver (red arrow in Fig. 5c): (a) during the preparative learning step, (b) over 100 dynamics acquisitions extracted from the interventional period.

\section{In-vivo heating study on a porcine kidney}

Fig. 6 shows an overlay of the temperature map after $60 \mathrm{~s}$ of sonication with $100 \mathrm{~W}$ accoustic power without and with adjustment of the HIFU focal point position with respect to the target position. A low SNR value of 6 was obtained due to the difficulty to perform an accurate MR receiver coil placement. The tracking algorithm (which used a translational motion model) remained locked-on the observed motion pattern over the entire duration of the sonication experiment without apparent failure. The temperature evolution

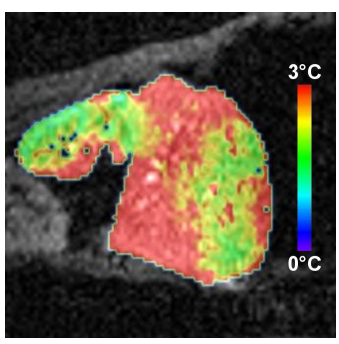

(a)

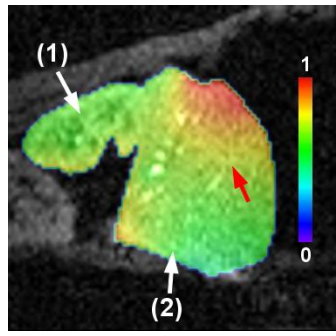

(c)

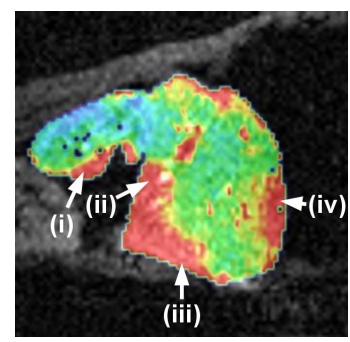

(b)

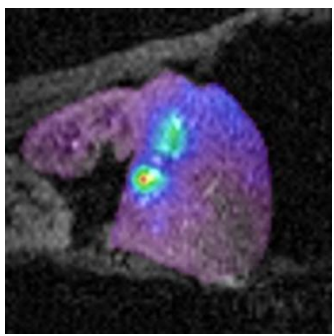

(d)
Fig. 5: Results obtained in the abdomen of a healthy volunteer (volunteer \#7 in Fig. 3): (a) and (b) show the temperature standard deviation map obtained with an affine motion model and the PCA-based motion descriptor, respectively. (c) and (d) display two PCA components $\left(B_{0}\right.$ and $\left.B_{3}\right)$ : For each pixel the amplitude of the displacement vector was computed, and each map was normalized between 0 and 1 for vizualisation. Results are presented in superimposition with the anatomical image depicting the kidney (arrow (1)) and the liver (arrow (2)).

of the voxel with the maximal temperature rise is plotted in Fig. 6c. However, the untracked experiment reached a lower final temperature than the experiment performed with full motion compensation (tracked: $15{ }^{\circ} \mathrm{C}$; untracked: $10{ }^{\circ} \mathrm{C}$ ). The estimated displacement was used off-line as reference to quantify the quality of the proposed PCA-registration. A signal decrease, correlated with the temperature rise could be observed in the heated area (see the time curve over the whole experiment of the estimated vertical displacement in a voxel located in the heated area of Fig. 7a). It can be observed in Fig. $7 \mathrm{a}$ and $7 \mathrm{~b}$ that only the first eigenvector visually matched properly the translational kidney displacement. While the first component of the motion descriptor visually described properly the motion pattern induced by the assisted respiration (see Fig. 7d), the second component was very noisy (see Fig. $7 \mathrm{f}$ ). The period of the respiratory cycle was $\approx 5$ seconds and the main peak located at $0.2 \mathrm{~Hz}$ on the spectral magnitude of the first component (see 7e). The ratio between the maximal spectral magnitude for frequencies lower and higher than 4 $\mathrm{Hz}$ was 0.03 for the first component, and reached 0.69 for the second (see Fig. 7e and 7g). The optimized size of the motion descriptor was thus of 1 component. Similarly to the ex-vivo experiment, the Cornelius\&Kanade approach shows a shift of the estimated displacement correlated with the magnitude signal decrease (see Fig. 7.a and 7.h). Using the reference translation displacement estimated on-line, we evaluated that this shift reached a maximal value of 1.5 millimeters at the 
end of the heating process. The PCA-based approach remained stable over the time since less than 0.1 millimeters of error was estimated.

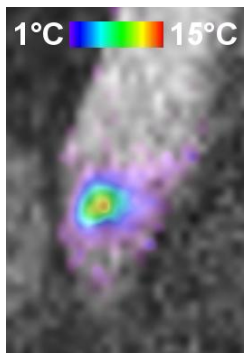

(a)

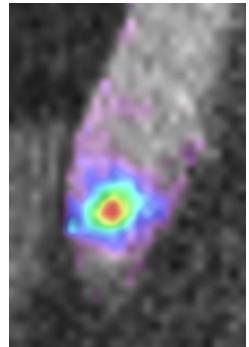

(b) (a)

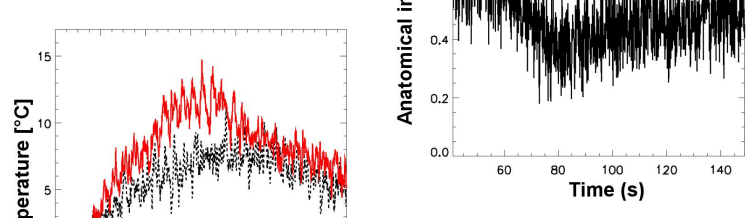

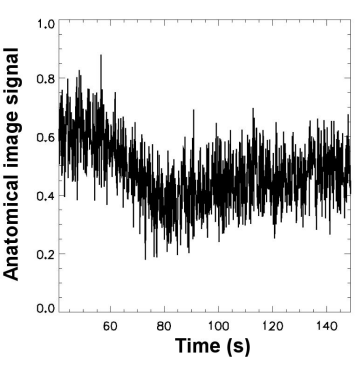

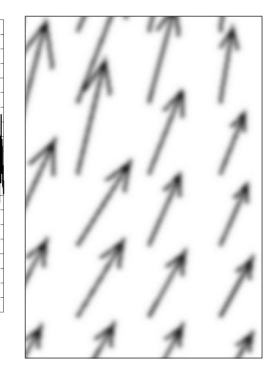

(b)

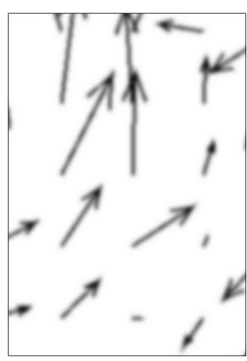

(c) (d)

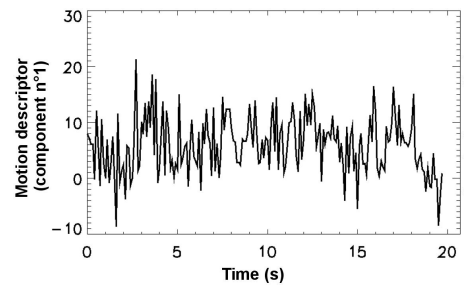

(f)

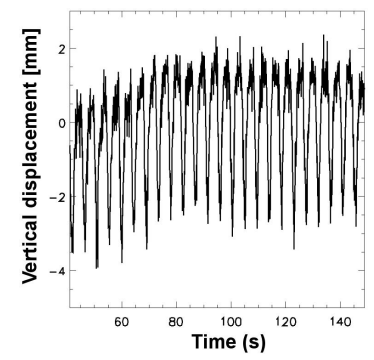

(h)

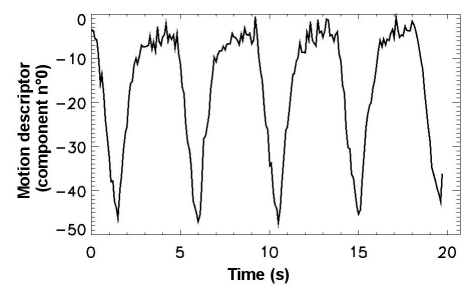

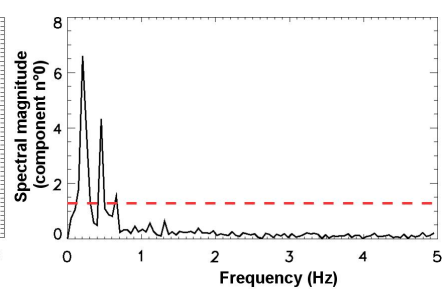

(e)

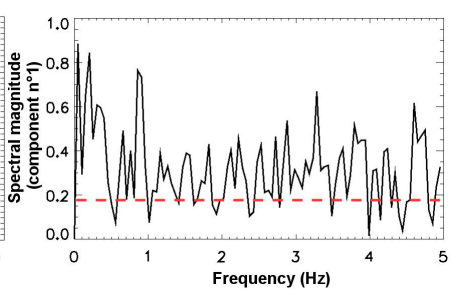

(g)

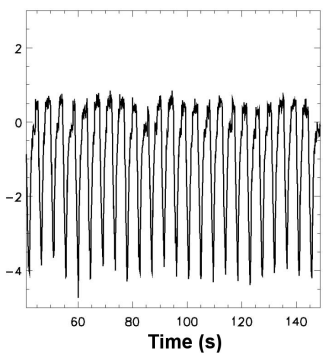

(i)
2) To discard during the hyperthermia procedure, possible local intensity perturbations induced by the heating: While purely optical-flow-based realignment may lead to temperature map computation errors for the case of local or global intensity changes, PCA-based realignment can give accurately registered temperature maps for the case of periodical motion, since it relies on a global fit of the principal components. Since the proposed method employs optical flow only during the preparative step and relies on a global fit of the image content during the intervention, estimated transformation $T_{t}$ was thus less susceptible to those local intensity variations.

It is important to note that the proposed motion estimation was not constrained to estimate positions present in the collection. The proposed approach assumes that the deformation must be properly described by a linear combination of the flow basis $P_{i}$. In the tested data, the proposed motion model was shown to be efficient to estimate organ motion amplitudes higher than the ones observed in the learning phase (see Fig. 4).

In addition, the method provided a characterization of the organ displacement which allowed, in turn, an accurate
Fig. 7: Motion estimation results otained for the in-vivo HIFU heating experiment. The temporal evolution of the magnitude signal in the voxel with the maximal temperature rise is shown in (a) (note the signal intensity loss during the heating process). The two first PCA components $\left(B_{0}\right.$ and $\left.B_{1}\right)$ are reported in (b) and (c), respectively. The temporal evolution of the two first coefficients of the PCA-base motion descriptor (i.e. $D_{0}$ and $\left.D_{1}\right)$ and their corresponding representation in the spectral domain are displayed in (d), (e), (f) and (g). The red dashed line in (e) and (g) shows the value of $20 \%$ of the main peak below $4 \mathrm{~Hz}$. The temporal evolution of the estimated displacement in the head-foot direction with the Cornelius\&Kanade algorithm and the PCA-based approach are displayed in (h) and (i), respectively.

modelling of the magnetic field perturbation. As a result, the PCA-based motion descriptor improved the precision of the temperature computation, compared to an affine motion model. The proposed approach also allowed to extrapolate motion amplitudes higher than the ones observed in the 
preparative learning step and could still provide an estimate of the reference phase in those cases. It is interesting to note that few temperature precision improvements were obtained on patients depicting small motion amplitude variation between the preparative learning step and the hyperthermia procedure (see volunteers \#2, \#3, \#5 and \#10 in Fig. 3). Although an affine motion model was found to perform well in many patients, the PCA-based approach should be preferred to guarantee good treatment in such outlier cases. The method provided thermal maps registered to a reference position, which is a necessary prerequisite for the computation of the thermal dose [3], and thus for the on-line assessment of the progress of the interventional procedure. It must be emphasized that, in the current study, the magnetic field perturbation was estimated assuming a simple linear magnetic field variation with organ displacement. Although this assumption holds in general for small displacements, the precision of this simple model must be carefully evaluated in regions displaying large motion amplitudes or large susceptibility variations, such as in the vicinity of the digestive tubes (see arrow (i) in Fig. 3c), of the quadratus lumborum muscle (see arrow (ii) in Fig. 3c), of the vertebral column (see arrow (iii) in Fig. 3c), and in the upper part of the liver (see arrow (iv) in Fig. 3c). This can in practice be achieved by mapping the fitting error from Eq. (4). This also allows to discard regions where low signal levels in conjunction with large susceptibility variations prevent to adjust successfully the linear model to the phase data.

The determination of the size $M$ of the subset of principal components was a key point. In all presented experiments, the energy of the first eigenvalue was higher than $99 \%$ of the total energy. This can be attributed to the fact that motion of abdominal organs is dominated by linear shifts due to the displacement of the diaphragm during the respiratory cycle. The determination of $M$ could thus not be based on a pure statistical analysis. The proposed criterion based on the Fourier representation of each component of the motion descriptor allowed to discriminate physiological and noise contributions: An optimal size of 1 was determined for the in-vivo porcine kidney experiment which matched the kidney displacement with the used imaging plane orientation. A minimal size of 5 was required in the human study, since the liver depicts a complex deformation together with arterial pulsations. Althought a size higher than 6 would have been set in 6 cases with the proposed criterion (note that this provided improved thermometry results in Fig. 3), we limited this value to 6 for computational time reasons.

Ries et al. have shown that, for typical abdominal organ motion, a temperature elevation comparable to static control experiments can be achieved for update frequencies of more than $10 \mathrm{~Hz}$ and latencies of $100 \mathrm{~ms}$ [13]. On our test platform, the latency was composed as the sum of: the remaining acquisition time after echo-formation $(\approx 50 \mathrm{~ms})$, the required data transport time $(\approx 10 \mathrm{~ms})$, the image processing time, and the switching time of the HIFU-generator $(\approx 10$ $\mathrm{ms})$. Thus, computation time for the whole image processing must typically be below $30 \mathrm{~ms}$ to be compatible with the real-time constraint. The PCA-based image realignment has computational advantages: the reduction of complex periodic motion patterns to the most significant principal components minimizes the degrees of freedom for the registration without a priori assumptions or simplifications of the form of the motion. On our implementation, the computation time required for the processing of one image was below $30 \mathrm{~ms}$. Depending on the displacement amplitude of the target, between 8 and $25 \mathrm{~ms}$ were required for the motion estimation process. Less than 1 ms was necessary for the calculation of the motion corrected temperature map. This indicates that the proposed method is suitable for real-time interventional MR-guidance, as shown in the in-vivo heating study.

Although the proposed method improves previously suggested approaches for MR-guided thermotherapy of mobile organs, several limitations remain. The first limitation is the additional scan required to learn the motion pattern before hyperthermia. However, in practice this limitation is less severe, since the proposed correction approach is paired with high frame rate imaging and thus a re-calibration can be completed in the relatively short time of two to five respiratory cycles. The second problem arises when new positions are observed during hyperthermia, for which the learned motion pattern is inadequate. In this case, the proposed approach must be paired with a correction adapted for accidental motion, as detailed in [12]. Finally, the effect of through plane motion is a severely limiting factor. In the scope of the presented study, this has been avoided by aligning the slice parallel to the main direction of motion. Since real-time acquired MR-images have generally an anisotropic image resolution (roughly 2-3 times higher in the slice than perpendicular to the slice) this did in none of the volunteers lead to severe problems due to residual through-slice motion. However, this is an inconvenient limitation of the degree of freedom for slice positioning (which for diagnostic reasons might be unfavorable) and complicates the slice placement in the planning of the intervention. Furthermore, it can not be excluded that patients with liver pathologies display more complex 3D motion patterns than the examined cases. In such cases the only true remedy would be to acquire extended 3D data volumes and apply the proposed methods in 3D. However, although it is well established that MR-imaging can provide motion estimates with a high spatial resolution, it is difficult to acquire on-line 3D isotropic images because technical limitations lead to a compromize between spatial and temporal resolution and low SNR associated with fast 3D acquisition sequences. Alternative approaches, based on the use of additional informations such as navigator echoes, in combination with adaptive slice tracking to address 3D trajectories, may be used [13].

\section{CONCLUSION}

The proposed approach addresses both motion compensated MR thermometry and target tracking by applying high frame rate MRI coupled with a real-time motion estimation and characterization obtained from all incoming images. The PCAbased motion descriptor introduced in [16] and [17] presented several advantages compared to previously suggested approaches: the image-based estimated motion was robust with respect to local and global intensity changes, and artifact-free 
temperature maps were obtained in all examined cases. The method allowed to achieve a sub-second temporal resolution with very short image latencies over sustained imaging periods of several minutes. During the intervention both, the target location and the target temperature were continuously available with a high temporal resolution and precision.

This renders the method well suitable for the MR-guidance of a heating intervention on abdominal organs in vivo under free-breathing over sustained periods of several minutes and presents therefore a step towards clinical non-invasive HIFU therapies of kidney and liver tumors.

\section{ACKNOWLEDGMENT}

The authors would like to thank the "Ligue Nationale Contre le Cancer", the "Conseil Régional d'Aquitaine", "Diagnostic Molecular Imaging” EC-FP6-project LSHB-CT-2005-512146, the "Agence Nationale de la Recherche" (project "MRgHIFUALKT"), Foundation InNaBioSanté (project ULTRAFITT) and Philips Healthcare.

\section{REFERENCES}

[1] H. E. Cline, J. F. Schenck, K. Hynynen, R. D. Watkins, S. P. Souza, and F. A. Jolesz, "MR-guided focused ultrasound surgery," J Comput Assist Tomogr, vol. 16, no. 6, pp. 956-965, 1992.

[2] M. Pernot, J. F. Aubry, M. Tanter, J. L. Thomas, and F. M., "High power transcranial beam steering for ultrasonic brain therapy," Physics in Medicine and Biology, vol. 48, pp. 2577-2589, 2003.

[3] S. A. Sapareto and W. C. L. Dewey, "Thermal dose determination in cancer therapy," Int. J. Radiation Oncology Biol. Phys., vol. 10, pp. 787-800, 1984.

[4] B. Quesson, J. A. de Zwart, and C. T. W. Moonen, "Magnetic resonance temperature imaging for guidance of thermotherapy," Magnetic Resonance in Medicine, vol. 12, pp. 525-533, 2000.

[5] R. D. Peters, R. Hinks, and R. M. Henkelman, "Ex vivo tissue-type independence in proton-resonance frequency shift mr thermometry," Magnetic Resonance in Medicine, vol. 40, pp. 454-459, 1998.

[6] J. De Poorter, C. De Wagter, Y. De Deene, C. Thomson, F. Stahlberg, and E. Achten, "The proton resonance frequency shift method compared with molecular diffusion for quantitative measurement of two dimensional time dependent temperature distribution in phantom," Journal of Magnetic Resonance Imaging, vol. 103, pp. 234-241, 1994.

[7] B. Denis de Senneville, C. Mougenot, and C. T. W. Moonen, "Real time adaptive methods for treatment of mobile organs by MRI controlled high intensity focused ultrasound," Magnetic Resonance in Medicine, vol. 57, no. 2, pp. 319-30, 2007.

[8] J. Maintz and M. Viergever, "A survey of medical image registration," Med Image Anal, vol. 2, no. 1, pp. 1-36, 1998.

[9] I. Mikic, S. Krucinski, and T. J. D., "Segmentation and tracking in echocardiographic sequences: Active contours guided by optical flow estimates," Transactions on Medical Imaging, vol. 17, no. 2, pp. 274 284, 1998.

[10] J. A. de Zwart, F. C. Vimeux, J. Palussiere, R. Salomir, B. Quesson, C. Delalande, and C. T. W. Moonen, "On-line correction and visualization of motion during MRI-controlled hyperthermia," Magnetic Resonance in Medicine, vol. 45, no. 1, pp. 128-37, 2001.

[11] S. Roujol, M. Ries, B. Quesson, C. T. W. Moonen, and B. Denis de Senneville, "Real-time MR-thermometry and dosimetry for interventional guidance on abdominal organs," Magnetic Resonance in Medicine, vol. 63, no. 4, pp. 1080-7, 2010

[12] B. Denis de Senneville, S. Roujol, C. T. W. Moonen, and M. Ries, "Motion correction in MR thermometry of abdominal organs: A comparison of the referenceless vs the multi-baseline approach," Magnetic Resonance in Medicine, vol. 64(5), pp. 1373-1381, 2010.

[13] M. Ries, B. Denis de Senneville, S. Roujol, Y. Berber, B. Quesson, and C. T. W. Moonen, "Real-time 3D target tracking in MRI guided focused ultrasound ablations in moving tissues," Magnetic Resonance in Medecine, vol. 64, no. 6, pp. 1704-12, 2010.
[14] M. J. Black, Y. Yacoob, A. D. Jepson, and D. J. Fleet, "Learning parameterized models of image motion," IEEE Proc. Computer Vision and Pattern Recognition, pp. 561-567, 1997.

[15] F. Mauldin, F. Viola, and W. Walker, "Complex principal components for robust motion estimation," IEEE Trans Ultrason Ferroelectr Freq Control, vol. 57(11), pp. 2437-49, 2010.

[16] G. Maclair, B. Denis de Senneville, M. Ries, B. Quesson, P. Desbarats, J. Benois-Pineau, and C. T. W. Moonen, "PCA-based image registration : Application to on-line mr temperature monitoring of moving tissues," in ICIP, vol. III. IEEE, 2007, pp. 141-144.

[17] _ , "PCA-based magnetic field modeling : Application for on-line $\mathrm{mr}$ temperature monitoring," in MICCAI, 2007, pp. 411-419.

[18] S. J. Graham, M. J. Bronskill, and R. M. Henkelman, "Time and temperature dependence of MR parameters during thermal coagulation of ex vivo rabbit muscle," Magnetic Resonance in Medecine, vol. 39(2), pp. 198-203, 1998.

[19] K. J. Friston, J. Ashburner, C. D. Frith, J. B. Poline, J. D. Heather, and R. S. J. Frackowiak, "Spatial registration and normalisation of images," Human Brain Mapping, vol. 2, pp. 165-189, 1995.

[20] B. K. P. Horn and B. G. Schunck, "Determining optical flow," Artificial intelligence, vol. 17, pp. 185-203, 1981.

[21] N. Cornelius and T. Kanade, "Adapting optical flow to measure object motion in reflectance and x-ray image sequences," ACM SIGGRAPH/SIGART Interdisciplinary Workshop on motion: representation and perception, Toronto, Canada, vol. 15, pp. 420-437, 1983.

[22] I. Pratikakis, C. Barillot, P. Hellier, and E. Mmin, "Robust multiscale deformable registration of 3D ultrasound images," International Journal of Image and Graphics, vol. 3, no. 4, pp. 547-566, 2003.

[23] D. Marquardt, "An algorithm for least squares estimation on nonlinear parameters," SIAM J. APPL. MATH, vol. 11, pp. 431-441, 1963. 